Computer Physics Communications 16 (1979) 243-265

(c) North-Holland Publishing Company

\title{
ALFVEN - A TWO-DIMENSIONAL CODE BASED ON SHASTA, SOLVING THE RADIATIVE, DIFFUSIVE MHD EQUATIONS
}

\author{
Wim J. WEBER * \\ Sterrekundig Instituut, Zonnenburg 2, Utrecht, The Netherlands \\ and \\ J.P. BORIS and J.H. GARDNER \\ Naval Research Laboratory, Washington DC, USA
}

Received 23 May 1978

\section{PROGRAM SUMMARY}

Title of program: ALFVEN

Catalogue number: ABUX

$\begin{array}{ll}\text { Computer } & \text { Installation } \\ \text { CDC } 6500 & \text { ACCU, Utrecht } \\ \text { CDC } 6600 & \text { Computer Centre, Groningen } \\ \text { IBM 370/168 } & \text { CECAM, Orsay, France } \\ \text { IBM 370/158 } & \text { CRI, Leiden }\end{array}$

Operating system: SCOPE 3.4 resp. OS-MVT or OS-MVS

Program language used: FORTRAN IV

High speed storage required: 55 kwords (CDC), 300 kbytes (IBM)

No. of bits in a word: 60 (CDC), 32 (IBM), single precision

Overlay structure: none

No. of magnetic tapes: none

* Presently at The Auroral Observatory, PB 953, 9001 Tromsö, Norway.

\begin{abstract}
Other peripherials used: card reader, line printer, magnetic disks
\end{abstract}

No. of cards in combined program and test deck: 2600

Keywords: Diffusive, radiative MHD, 2-dim, second-order accuracy, explicit convection, implicit diffusion, monotonic solutions, SHASTA numerical scheme, low-diffusion scheme

\section{Nature of physical problem}

A two-dimensional computer program to be used for solving hydrodynamic and hydromagnetic equations in various geometries $[1,2]$. The effects of gravity, ohmic dissipation and -diffusion, radiative losses and thermal conduction are included through an implicit, time-split coupled, step. The convective part of the equations is solved by the SHASTA numerical code [3-7], having second-order accuracy but guaranteeing monotonicity in the directly-convected quantities. One-dimensional and isothermal applications are possible.

The program uses the equation for conservation of total energy ( $U$-version).

\section{Method of solution}

Vectorized, two-dimensional explicit convection step with predictor-corrector ordening for second-order solution with removal of numerically produced extrema, time-split coupled to an implicit, tridiagonal matrix solver for physical conduction, radiation and diffusion [8]. 


\section{Typical running time}

Achicving equilibrium in longest physical time scale will require typically 1200 time steps. Duration of one time step ( $-5 \%$ for adiabatic, $-25 \%$ for diffusion free calculations): $22 \mathrm{~s}($ CDC 6500), $6 \mathrm{~s}$ (CDC 6600), 4 s (IBM 370/168),

$15 \mathrm{~s}$ (IBM $370 / 158$ ) for a $63 \times 43 \mathrm{grid}$ in single precision.

References

[1] K.V. Roberts and D.E. Potter, Magnetohydrodynamic calculations, in Methods in computational physics, vol. 9, eds. Alder, Fernbach and Rotenberg (Academic Press, New York, London, 1970) p. 339.

[2] W.J. Weber, Thesis, University of Utrecht (1978).

[3] J.P. Boris and D.L. Book, J. Comput. Phys. 11 (1973) 38.
[4] J.P. Boris, SHAS2D - A fully compressible hydrody namics code in 2 dimensions, NRL Memorandum Report 2542, Washington (1972).

[5] D.L. Book, J.P. Boris and K. Hain, J. Comput. Phys. 18 (1975) 248.

[6] J.P. Boris and D.L. Book, J. Comput. Phys. 20 (1976) 397.

[7] J.P. Boris, Flux-corrected transport modules for solving generalized continuity equations, NRL Memorandum Report 3237, Washington (1976).

[8] S.I. Braginskii, Rev. Plasma Phys. 1, Consultants Bureau, New York (1965). 